
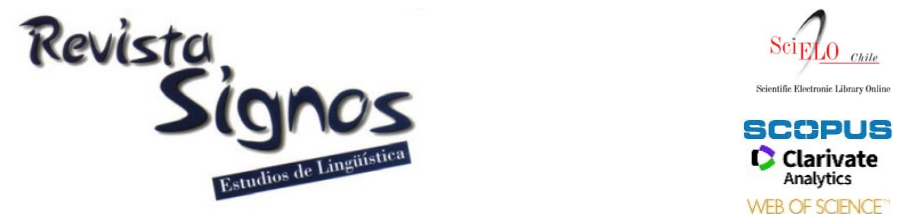

\title{
Es la más mejor. Sobre la lexicalización de los comparativos sintéticos como adjetivos positivos en español actual
}

\section{Es la más mejor. On lexicalization of synthetic comparatives as positive adjectives in Modern Spanish}

\author{
Elena Felíu Arquiola \\ UNIVERSIDAD DE JAÉN \\ ESPAÑA \\ efeliu@ujaen.es
}

\author{
Enrique Pato \\ UNIVERSITÉ DE MONTRÉAL \\ CANADÁ \\ enrique.pato-maldonado@umontreal.ca
}

Recibido: 04-III-2020 / Aceptado: 05-VIII-2020

DOI: $10.4067 /$ S0718-09342021000200332

\section{Resumen}

En este trabajo de corte cuantitativo se analiza el comportamiento de los comparativos sintéticos del español cuando aparecen precedidos de los cuantificadores 'más' y 'menos'. En concreto, a partir de los datos obtenidos del Corpus del español: Web/Dialectos, se da a conocer su distribución geográfica actual, se determina qué formas son las empleadas (y con qué frecuencia), en qué contextos sintácticodiscursivos aparecen y cómo se perfila su proceso de positivización. Estas combinaciones (documentadas desde antiguo) no son exclusivas de España, sino que se emplean también plenamente en las variedades americanas. Las pruebas que muestran el proceso de 'positivización' son varias: los comparativos sintéticos se documentan combinados con el modificador de grado 'muy' ('muy mayor'/ 'menor'/ 'mejor'/ 'peor'); admiten la derivación en '-mente' ('mejormente', 'peormente', 'mayormente', 'menormente'), así como la coordinación con otro adjetivo en construcción comparativa ('más mejor y más larga'). Además, mostramos que la lexicalización de 'mayor' como adjetivo positivo no solo se da cuando este adjetivo presenta significado temporal ('persona más mayor'), sino que también se registra con un significado de dimensión física real o figurada ('los más mayores yacimientos de cobre'). Lo mismo encontramos para el caso de 'menor', que presenta un sentido temporal ('el más menor de sus hijos') y otro de dimensión física ('delitos más menores'). Por lo que respecta a 'mejor' y 'peor', el primero se estaría lexicalizando con el significado de 'más bueno/ bien' ('los más mejores ladrones') y el segundo con el de 'más malo/ mal' ('el menos peor de los escenarios').

Palabras Clave: Español actual, gramática, variación, adjetivos comparativos, lexicalización. 


\begin{abstract}
This paper takes a quantitative approach to analyzing the behavior of Spanish synthetic comparatives when they are preceded by the quantifiers más and menos. In particular, taking the data obtained from the Corpus del español: Web/Dialectos, we give an account of their current geographical distribution, as well as we determine what forms are used (and how often), in what syntactic-discursive contexts they appear and how their positivization process is outlined. These combinations (documented since ancient times) are not exclusive to Spain, but are also used in some American Spanish varieties. Several tests support the process of 'positivization': the synthetic comparatives are documented combined with the degree modifier muy (muy mayor/ menor/ mejor/ peor); they allow -mente derivation (mejormente, peormente, mayormente, menormente), as well as coordination with another adjective in comparative constructions (más mejor y más larga). In addition, we show that the lexicalization of mayor as a positive adjective does not only take place when this adjective has a temporal meaning (persona más mayor), but also when its meaning relates to real or figurative physical dimension (los más mayores yacimientos de cobre). The same thing is found in menor, which presents both a temporal meaning (el más menor de sus hijos) and a physical dimension meaning (delitos más menores). As for mejor and peor, the former would be undergoing a lexicalization process with the meaning 'more good/ well' (los más mejores ladrones), while the latter a process with the meaning of 'more bad/ badly' (el menos peor de los escenarios).
\end{abstract}

Key Words: Modern Spanish, grammar, variation, comparative adjectives, lexicalization.

\title{
INTRODUCCIÓN
}

Como es sabido, los comparativos sintéticos (también llamados sincréticos o comparativos en -or) 'mejor', 'peor', 'mayor' y 'menor' no requieren su combinación con cuantificadores comparativos (*más mejor, *menos peor), ya que al ser -por naturaleza- elementos comparativos de superioridad "envuelven el adverbio "más" (Bello, 1847; Gómez Torrego, 1998; RAE/ASALE, 2009: 2288). Por otro lado, el Diccionario panhispánico de dudas (RAE/ASALE, 2005) nos recuerda que el adverbio 'más' se combina con adjetivos positivos que denotan propiedades graduables ('más alto', 'más fuerte'; pero *más caduco, *más inmortal) y que no debe emplearse ante adjetivos superlativos (*más importantísimo) ni comparativos (*más mejor), salvo como veremos- en el caso de 'mayor' cuando funciona como adjetivo positivo. En general (Seco \& Hernández, 1999; Seco, 2000; entre otros), las construcciones 'más mejor' (por 'mejor'), 'más peor' (por 'peor') y 'más mayor' (por 'mayor') se han tratado tradicionalmente como redundancias vulgares, usos relegados "a los iletrados en el uso popular y rústico general" (Kany, 1969: 71). También son uno de los errores más comunes que cometen los aprendices de español como lengua extranjera (Robles Ávila, 1998).

Según la Nueva gramática de la lengua española (RAE/ASALE, 2009), la combinación de comparativos léxicos con cuantificadores comparativos se registra 'en la lengua 
rural de muchos países hispanohablantes', y el caso concreto de 'más mejor', 'en la lengua popular de algunos países' (sin entrar en mayor especificación); usos que se recomienda evitar en los registros formales.

La revisión de la bibliografía sobre este fenómeno permite observar, asimismo, que las descripciones previas han olvidado otras posibles combinaciones comparativas como 'más menor', 'menos mejor', 'menos peor', 'menos mayor' y 'menos menor'. Por otro lado, en los casos en que 'mayor' significa 'adulto; de edad avanzada; anciano', esto es, cuando se emplea como adjetivo del campo de la edad, ha dejado de ser adjetivo comparativo relacionado con la expresión del tamaño para pasar a ser adjetivo positivo de significado temporal (Madero Kondrat, 1983; Vigara Tauste, 2010, 2011). Con este sentido, que se atestigua ya en el trabajo de Covarrubias (1611), 'mayor' admite intensificación ('muy mayor'), superlación absoluta ('mayorcísimo'), relativa ('el más mayor') y gradación comparativa ('tan/ más/ menos mayor que...'). Creemos que este mismo proceso descrito y estudiado para el caso de 'mayor' -de comparativo sincrético a adjetivo positivo- está sucediendo con la forma 'menor', cuando presenta el significado temporal 'que no ha alcanzado la edad adulta; pequeño', cambio que se circunscribe también al campo de la edad, tal y como mostraremos en este trabajo. Un proceso similar parece estarse produciendo también en el caso de 'mejor' y 'peor', como veremos.

Con todo, aunque están proscritas en la gramática normativa (y no se enseñan de manera formal), estas expresiones analíticas ameritan un trabajo independiente, por varios motivos: en primer lugar, para conocer la distribución geográfica que alcanzan en español actual; en segundo lugar, para determinar qué formas son las empleadas y en qué porcentaje, tanto en singular ('más mejor') como en plural ('menos peores'); y, en tercer lugar, para saber en qué contextos aparece este uso. Teniendo en cuenta estos tres objetivos generales, el presente trabajo se estructura de la siguiente manera: tras esta breve introducción, damos a conocer los nuevos datos obtenidos en nuestra investigación en cuanto al uso, extensión y distribución geográfica del fenómeno (ver apartado 2), para después presentar su análisis e interpretación, donde se incluye la revisión de los contextos sintáctico-discursivos (sección 2.1), el proceso general de 'positivización' de los adjetivos comparativos en español actual (sección 2.2) y el estudio de cada par de comparativos sincréticos de forma independiente ('mayor' y 'menor' en apartado 2.3, 'mejor' y 'peor' en sección 2.4). Por último, se resumen las principales conclusiones alcanzadas y se presentan unas consideraciones finales.

\section{Nuevos datos}

Antes de presentar los datos de nuestra investigación hay que recordar que estas combinaciones se documentan ya en el mismo latín: magis melior (Vitruvio), magis maiores (magis maiores nugas egerit 'debería perderse las bromas más mayores/divertidas' 
(Plauto, Menaechmi, 55)), donde magis modifica al adjetivo comparativo en -ior (MeyerLübke, 1900; Grandgent, 1970; Vigara Tauste, 2010).

Además, por lo que respecta al caso del español, el fenómeno ha tenido continuidad en todas las épocas, pues 'más mejor' y 'más peor' se registran desde antiguo (García de Diego, 1970; Romero Cambrón, 1998), tal y como muestran los siguientes ejemplos procedentes de textos lingüísticamente fiables, es decir, testimonios originales, sin problemas de grafía, datación ni reconstrucción editorial (Rodríguez Molina \& Octavio de Toledo y Huerta, 2017, para el caso de los textos medievales) y albergados en el CORDE (RAE):

(1) a. E lo peor que puede seer en esta signification \& en estas costellationes; es que sea Saturno alçado en el medio cielo \& la luna en el ascendente recibiendo su friura de Saturno. \& su sequedat $\&$ su infortuna $\&$ sus malas naturas que con esto sera la cosa mas peor \& mas afincada (Alfonso X, Judizios de las estrellas, 1254-1260, CORDE).

b. E deuedes saber que el arena cauadiça si qujer en las cubiertas de las camaras sy qujer en los paujmjentos \& en las paredes es mas mejor que la otra (Ferrer Sayol, Libro de Palladio, 1380-1385, CORDE).

c. Pregúntoos agora yo, amigos, pues estáys en reputación de sabios: ¿quál fuera mejor o, por mejor dezir, quál fuera menos peor: que este philósopho fuera hombre simple y de buena vida, o ser como fue de alta eloqüencia y de mala fama? (Fray Antonio de Guevara, Reloj de Príncipes, 1529-1531, CORDE).

d. Favorezcame Maria,/ y los Angeles del Cielo,/ y tambien me favorezca/ la Virgen del Buen Suceso./ Nuestra Señora del Risco/ tambien en mi amparo quiero,/ que es la Virgen mas mejor,/ que veneran nuestros Reynos (José F. de Isla, Descripción de la máscara o mojiganga, 1787, CORDE).

El corpus empleado en esta investigación, centrada en el español actual, es el Corpus del español: Web/Dialectos, base de datos que alberga más de dos mil cien millones de palabras procedentes de páginas web de los 21 países de habla hispana publicadas entre 2013 y 2014. Las búsquedas de las formas, en todos los países, se realizaron entre los meses de agosto y octubre de 2018. A este respecto, hay que matizar una serie de hechos. En primer lugar, dado que 'más mayor' es una construcción admitida ya por la academia (Mi hermano Pedro es más mayor que tû́), no formó parte de las búsquedas. Por otro lado, dejamos también fuera de este estudio los casos de 'cuanto' / 'contra'/ 'mientras más mejor 'y los casos de 'por lo menos'/ 'al menos, mejor que'..., es decir, las oraciones comparativas proporcionales o correlativas. Por lo que respecta a los ejemplos que presentamos, mantenemos la grafía original que figura en el corpus en todos los casos e indicamos el país de origen entre paréntesis. 
Las siguientes tablas resumen los datos obtenidos (912 en total) para 'más mejor'/ 'peor'/ 'menor' en singular (363 casos, Tabla 1), 'más mejores' / 'peores' / 'menores en plural' (78 casos, Tabla 2), 'menos mejor'/ 'peor'/ 'menor' en singular (433 casos, Tabla 3) y 'menos mejores'/ 'peores'/ 'menores' en plural (38 casos, Tabla 4). Los resultados indican que dos son las construcciones con un mayor empleo: 'más mejor' (280 casos, 77,13\%) y 'menos peor' (406 casos, 93,76\%). Sin embargo, aunque en menor medida, también es posible documentar las demás combinaciones².

Conozcamos con detalle los datos obtenidos en el corpus empleado mediante las siguientes tablas. En la Tabla 1, a continuación, presentamos los datos relativos al empleo en singular de 'más mejor', 'más peor 'y 'más menor'.

Tabla 1. Número de casos (N) y porcentajes (\%) de 'más mejor'/ 'peor'/ 'mayor'/ 'menor' por países.

\begin{tabular}{|l|c|c|c|c|c|c|c|c|}
\hline \multirow{2}{*}{\multicolumn{1}{c|}{ País }} & \multicolumn{2}{c|}{ más mejor } & \multicolumn{2}{c|}{ más peor } & \multicolumn{2}{c|}{ más menor } & \multicolumn{2}{c|}{ Totales } \\
\cline { 2 - 9 } & $\mathbf{N}$ & $\mathbf{\%}$ & $\mathbf{N}$ & $\mathbf{\%}$ & $\mathbf{N}$ & $\mathbf{\%}$ & $\mathbf{N}$ & $\mathbf{\%}$ \\
\hline España & 101 & 82,11 & 21 & 17,07 & 1 & 0,81 & 123 & 33,88 \\
\hline México & 31 & 67,39 & 13 & 28,26 & 2 & 4,34 & 46 & 12,67 \\
\hline Argentina & 27 & 75 & 9 & 25 & 0 & - & 36 & 9,91 \\
\hline Estados Unidos & 26 & 92,85 & 2 & 7,14 & 0 & - & 28 & 7,71 \\
\hline Chile & 21 & 80,76 & 5 & 19,23 & 0 & - & 26 & 7,16 \\
\hline Perú & 15 & 78,94 & 3 & 15,78 & 1 & 5,26 & 19 & 5,23 \\
\hline Venezuela & 10 & 66,67 & 4 & 26,67 & 1 & 6,66 & 15 & 4,13 \\
\hline Paraguay & 10 & 71,42 & 3 & 21,42 & 1 & 7,14 & 14 & 3,85 \\
\hline Colombia & 7 & 77,78 & 2 & 22,22 & 0 & - & 9 & 2,47 \\
\hline El Salvador & 5 & 62,5 & 2 & 25 & 1 & 12,5 & 8 & 2,2 \\
\hline Nicaragua & 5 & 71,42 & 2 & 28,57 & 0 & - & 7 & 1,92 \\
\hline Uruguay & 3 & 60 & 1 & 20 & 1 & 20 & 5 & 1,37 \\
\hline Bolivia & 2 & 40 & 3 & 60 & 0 & - & 5 & 1,37 \\
\hline Costa Rica & 5 & 100 & 0 & - & 0 & - & 5 & 1,37 \\
\hline Honduras & 4 & 100 & 0 & - & 0 & - & 4 & 1,1 \\
\hline Puerto Rico & 3 & 75 & 0 & - & 1 & 25 & 4 & 1,1 \\
\hline Ecuador & 2 & 50 & 2 & 50 & 0 & - & 4 & 1,1 \\
\hline Cuba & 2 & 66,67 & 1 & 33,33 & 0 & - & 3 & 0,82 \\
\hline Guatemala & 1 & 100 & 0 & - & 0 & - & 1 & 0,27 \\
\hline Rep. Dominicana & 0 & - & 1 & 100 & 0 & - & 1 & 0,27 \\
\hline Panamá & 0 & - & 0 & - & 0 & - & 0 & 0 \\
\hline Totales & $\mathbf{2 8 0}$ & 77,13 & $\mathbf{7 4}$ & 20,39 & $\mathbf{9}$ & 2,48 & $\underline{\mathbf{3 6 3}}$ & 100 \\
\hline
\end{tabular}

El número total de casos registrados de 'más' + comparativo sintético en singular es de 363. La combinación 'más mejor' alcanza un 77,13\% (280/363), seguida de 'más peor' $(20,39 \%, 74 / 363)$ y 'más menor' $(2,48 \%, 9 / 363)$. El Anova efectuado con los totales indica que los datos son altamente significativos $(F=7,5082, p=0,009$ y valor crítico de $F=4,0847)^{3}$.

Por lo que respecta al número de casos, aunque España es el país en el que más ejemplos se documentan (101 en total), los porcentajes muestran que no lidera ninguno de los usos. En efecto, para la construcción 'más mejor', otros países -con 5 o 
más ejemplos documentados- alcanzan porcentajes más elevados: es el caso de Costa Rica $(100 \%, 5 / 5)$ y Estados Unidos (92,85 \%, 26/28). Por lo que respecta a 'más peor', México (28,26\%, 13/46), Argentina (25\%, 9/36), Chile (19,23\%, 5/26) y España $(17,07 \%, 21 / 123)$ son los países con mayor porcentaje de aparición. Los datos alcanzados son de interés para la correcta descripción de este uso, ya que, según la RAE/ASALE (2009), se registra en el español popular y rural de España y 'raramente en América'.

Presentamos, seguidamente, la Tabla 2, en la que se recogen los datos relacionados con el empleo en plural de estas combinaciones ('más mejores'/ 'peores'/ 'menores') como adjetivos.

Tabla 2. Número de casos (N) y porcentajes (\%) de 'más mejores'/ 'peores' / 'menores' por países.

\begin{tabular}{|l|c|c|c|c|c|c|c|c|}
\hline \multirow{2}{*}{\multicolumn{1}{|c|}{ País }} & \multicolumn{2}{|c|}{ más mejores } & \multicolumn{2}{c|}{ más peores } & \multicolumn{2}{c|}{ más menores } & \multicolumn{2}{c|}{ Totales } \\
\cline { 2 - 10 } & $\mathbf{N}$ & $\mathbf{\%}$ & $\mathbf{N}$ & $\mathbf{\%}$ & $\mathbf{N}$ & $\mathbf{\%}$ & $\mathbf{N}$ & $\mathbf{\%}$ \\
\hline España & 20 & 80 & 3 & 12 & 2 & 8 & 25 & 29,3 \\
\hline Estados Unidos & 8 & 100 & 0 & - & 0 & - & 8 & 10,1 \\
\hline Argentina & 5 & 62,5 & 0 & - & 3 & 37,5 & 8 & 9,09 \\
\hline México & 3 & 50 & 2 & 33,33 & 1 & 16,67 & 6 & 12,12 \\
\hline Chile & 6 & 100 & 0 & - & 0 & - & 6 & 6,06 \\
\hline Perú & 4 & 100 & 0 & - & 0 & - & 4 & 5,05 \\
\hline Cuba & 4 & 100 & 0 & - & 0 & - & 4 & 4,04 \\
\hline Colombia & 2 & 66,67 & 1 & 33,33 & 0 & - & 3 & 2,02 \\
\hline Venezuela & 1 & 50 & 1 & 50 & 0 & - & 2 & 2,02 \\
\hline Bolivia & 1 & 50 & 1 & 50 & 0 & - & 2 & 2,02 \\
\hline Rep. Dominicana & 1 & 50 & 1 & 50 & 0 & - & 2 & 2,02 \\
\hline Guatemala & 0 & - & 2 & 100 & 0 & - & 2 & 5,05 \\
\hline Uruguay & 0 & - & 2 & 100 & 0 & - & 2 & 2,02 \\
\hline Nicaragua & 1 & 100 & 0 & - & 0 & - & 1 & 2,02 \\
\hline Costa Rica & 1 & 100 & 0 & - & 0 & - & 1 & 1,01 \\
\hline Ecuador & 1 & 100 & 0 & - & 0 & - & 1 & 1,01 \\
\hline Paraguay & 0 & - & 1 & 100 & 0 & - & 1 & 1,01 \\
\hline Panamá & 0 & - & 0 & - & 0 & - & 0 & - \\
\hline El Salvador & 0 & - & 0 & - & 0 & - & 0 & - \\
\hline Honduras & 0 & - & 0 & - & 0 & - & 0 & - \\
\hline Puerto Rico & 0 & - & 0 & - & 0 & - & 0 & - \\
\hline Totales & $\mathbf{5 8}$ & 74,36 & $\mathbf{1 4}$ & 17,94 & $\mathbf{6}$ & 7,7 & $\mathbf{7 8}$ & 100 \\
\hline
\end{tabular}

Por lo que respecta a las combinaciones en plural, el número total de ocurrencias llega a 78. La combinación 'más mejores' alcanza un 74,36\% (58/78), seguida de 'más peores' 17,94 \% (14/78) y 'más menores' 7,70\% (6/78). El Anova efectuado nos indica que los datos son estadísticamente significativos $(F=5,1546, p=0,02$ y valor crítico de $F=4,0847)$. Para 'más mejores' los países con mayor porcentaje de aparición son Estados Unidos (100\%, 8/8), Chile (100\% 6/6), España (80\%, 20/25) y Argentina $(62,50 \%, 5 / 8)$. 
En lo que respecta a los datos relativos a las combinaciones 'menos mejor' y 'menos peor', se presentan en la Tabla 3. La combinación 'menos menor' solo obtiene un caso en México; la falta de datos impide que figure en la tabla.

Tabla 3. Número de casos (N) y porcentajes (\%) de 'menos mejor'/ 'peor' por países.

\begin{tabular}{|l|c|c|c|c|c|c|}
\hline \multirow{2}{*}{\multicolumn{1}{|c|}{ País }} & \multicolumn{2}{|c|}{ menos mejor } & \multicolumn{2}{c|}{ menos peor } & \multicolumn{2}{c|}{ Totales } \\
\cline { 2 - 7 } & $\mathbf{N}$ & $\mathbf{0}$ & $\mathbf{N}$ & $\mathbf{\%}$ & $\mathbf{N}$ & $\mathbf{0}$ \\
\hline México & 2 & 2,12 & 92 & 97,88 & 94 & 21,7 \\
\hline Guatemala & 2 & 2,38 & 82 & 97,61 & 84 & 19,39 \\
\hline Argentina & 1 & 1,51 & 65 & 98,49 & 66 & 15,24 \\
\hline Colombia & 1 & 2,56 & 38 & 97,44 & 39 & 9 \\
\hline España & 8 & 27.58 & 21 & 72,42 & 29 & 8,75 \\
\hline El Salvador & 0 & - & 28 & 100 & 28 & 6,46 \\
\hline Estados Unidos & 0 & - & 20 & 100 & 20 & 4,61 \\
\hline Perú & 2 & 11,11 & 16 & 88,89 & 18 & 4,15 \\
\hline Venezuela & 6 & 46,15 & 7 & 53,85 & 13 & 3 \\
\hline Costa Rica & 0 & - & 8 & 100 & 8 & 1,84 \\
\hline Paraguay & 1 & 12,5 & 7 & 87,5 & 8 & 1,84 \\
\hline Honduras & 0 & - & 7 & 100 & 7 & 1,61 \\
\hline Ecuador & 0 & - & 5 & 100 & 5 & 1,15 \\
\hline Chile & 2 & 50 & 2 & 50 & 4 & 0,92 \\
\hline Uruguay & 1 & 33,33 & 2 & 66,67 & 3 & 0,69 \\
\hline Nicaragua & 1 & 50 & 1 & 50 & 2 & 0,46 \\
\hline Bolivia & 0 & - & 2 & 100 & 2 & 0,46 \\
\hline Cuba & 0 & - & 1 & 100 & 1 & 0,23 \\
\hline Puerto Rico & 0 & - & 1 & 100 & 1 & 0,23 \\
\hline Rep. Dominicana & 0 & - & 1 & 100 & 1 & 0,23 \\
\hline Panamá & 0 & - & 0 & - & 0 & 0 \\
\hline Totales & $\mathbf{2 7}$ & 6,24 & $\mathbf{4 0 6}$ & 93,76 & $\underline{\mathbf{4 3 3}}$ & 100 \\
\hline
\end{tabular}

En cuanto a 'menos' + comparativo sintético en singular, los casos llegan a 433. La combinación 'menos peor' alcanza un 93,76\% (406/433), seguida de 'menos mejor' 6,24\% (27/433). El Anova efectuado indica que los datos son estadísticamente muy significativos $(F=10,3649, p=0,0025$ y valor crítico de $F=4,0847)$.

La distribución de 'menos peor' alcanza porcentajes máximos en El Salvador, Estados Unidos, Costa Rica, Honduras y Ecuador (100\%), seguidos de Argentina (98,49\%, 65/66), México (97,98\%, 92/94), Guatemala (97,61\%, 82/84), Colombia (97,44\%, 38/39), Perú $(88,89 \%, 16 / 18)$ y Paraguay $(87,50 \%, 7 / 8)$. Con porcentajes medio-altos se sitúan España $(72,42 \%, 21 / 29)$ y Venezuela $(53,85 \%, 7 / 13)$. Para el caso de 'menos mejor' son también Venezuela 46,15\% (6/13) y España 27,58\% (8/29) los países que mayores porcentajes de aparición logran.

Finalmente, en la Tabla 4 presentamos los datos relativos al empleo de 'menos mejores' / 'peores'. Para el caso de 'menos menores' solo se registra un ejemplo en México, por ello tampoco figura en la Tabla. 
Tabla 4. Número de casos (N) y porcentajes (\%) de 'menos mejores'/ 'peores' por países.

\begin{tabular}{|l|c|c|c|c|c|c|}
\hline \multirow{2}{*}{\multicolumn{1}{|c|}{ País }} & \multicolumn{2}{|c|}{ menos mejores } & \multicolumn{2}{c|}{ menos peores } & \multicolumn{2}{c|}{ Totales } \\
\cline { 2 - 7 } & $\mathbf{N}$ & $\mathbf{\%}$ & $\mathbf{N}$ & $\mathbf{\%}$ & $\mathbf{N}$ & $\mathbf{0}$ \\
\hline México & 1 & 11,11 & 8 & 88,89 & 9 & 23,68 \\
\hline Colombia & 0 & - & 8 & 100 & 8 & 21,05 \\
\hline España & 3 & 75 & 1 & 25 & 4 & 10,52 \\
\hline Argentina & 1 & 33,33 & 2 & 66,67 & 3 & 7,89 \\
\hline Estados Unidos & 1 & 33,33 & 2 & 66,67 & 3 & 7,89 \\
\hline Bolivia & 0 & - & 2 & 100 & 2 & 5,26 \\
\hline El Salvador & 0 & - & 2 & 100 & 2 & 5,26 \\
\hline Guatemala & 0 & - & 2 & 100 & 2 & 5,26 \\
\hline Nicaragua & 0 & - & 2 & 100 & 2 & 5,26 \\
\hline Perú & 1 & 50 & 1 & 50 & 2 & 5,26 \\
\hline Paraguay & 0 & - & 0 & - & 1 & 2,63 \\
\hline Honduras & 0 & - & 1 & 100 & 1 & 2,63 \\
\hline Chile & 0 & - & 0 & - & 0 & - \\
\hline Costa Rica & 0 & - & 0 & - & 0 & - \\
\hline Cuba & 0 & - & 0 & - & 0 & - \\
\hline Ecuador & 0 & - & 0 & - & 0 & - \\
\hline Panamá & 0 & - & 0 & - & 0 & - \\
\hline Puerto Rico & 0 & - & 0 & - & 0 & - \\
\hline Rep. Dominicana & 0 & - & 0 & - & 0 & - \\
\hline Uruguay & 0 & - & 0 & - & 0 & - \\
\hline Venezuela & 0 & - & 0 & - & 0 & - \\
\hline Totales & $\mathbf{7}$ & 18,42 & $\mathbf{3 1}$ & 81,58 & $\underline{\mathbf{3 8}}$ & 100 \\
\hline
\end{tabular}

La documentación de 'menos' con adjetivos sintéticos en plural es la menos abundante de todas, con 38 casos en total, y solo se registra en algunos países de habla hispana. La combinación 'menos peores' alcanza un 81,58\% (31/38), seguida de 'menos mejores' 18,42\% (7/38). Como era de esperar, el Anova efectuado indica que los datos no son estadísticamente significativos $(F=2,4, p=0,129$ y valor crítico de $F$ $=4,0847)$, sin duda por el escaso número de ocurrencias documentadas en algunas de estas formas. Para el caso concreto de 'menos peores', dos países, Colombia (100\%, 8/8) y México (88,89\%,8/9), alcanzan los porcentajes más elevados.

\section{Análisis e interpretación}

Los datos que hemos presentado en el apartado 1 parecen indicar que no solo se emplea 'mayor' como adjetivo positivo en español actual, sino que este proceso de transformación de comparativo sincrético a adjetivo positivo ${ }^{4}$ se da también en el caso de 'mejor', 'peor' y 'menor'.

En lo que sigue revisaremos brevemente los contextos sintáctico-discursivos en los que suelen aparecer estas expresiones (sección 2.1), trataremos el proceso de 'positivización' de los adjetivos comparativos (sección 2.2) y nos centraremos en cada uno de los pares de comparativos sincréticos de forma independiente: 'mayor' y 'menor' (apartado 2.3); 'mejor' y 'peor' (sección 2.4). 


\subsection{Los contextos sintáctico-discursivos}

Varios son los contextos sintáctico-discursivos en los que las construcciones objeto de estudio suelen aparecer. Para ello, distinguiremos los casos en que estas combinaciones se forman en torno a un adjetivo de aquellos casos en que se forman en torno a un adverbio.

Trataremos en primer lugar los adjetivos 'mejor', 'peor' y 'menor'. Hay que señalar que la combinación con 'más' / 'menos' se da fundamentalmente en los siguientes contextos: a) en construcciones de superlativo relativo ('el'/ 'la' /'lo más mejor'/ 'peor'/ 'menor'), que constituyen el caso más frecuente; y b) en construcciones comparativas de superioridad ('más mejor'/ 'peor'/ 'menor que') y de inferioridad ('menos menor'/ 'peor'/ 'menor que').

El primero de los contextos que trataremos es el de las construcciones de superlativo relativo, que expresan "el grado máximo de alguna propiedad cuando se restringe a un conjunto delimitado de seres" (RAE/ASALE, 2009: 3432). Por una parte, podemos encontrar ejemplos -sin duda, los más abundantes- en los que no aparece un sustantivo explícito, en construcciones tradicionalmente conocidas como de 'sustantivación', según se observa en (2). Los tres primeros ejemplos constituyen casos de superlativo relativo sin coda superlativa (2a-c), mientras que los tres últimos sí presentan coda superlativa (2d-f):

(2) a. El peronismo funcionaría según ellos con esas categorías extremas y si lo contiene porque resultó el más mejor, adelante. Lo que siempre hay que preguntarse es que: -es mejor?, pero para qué? (Argentina).

b. con esfuerzo, dedicación y siguiendo algunas técnicas puedes descubrir la idea que puede ser la más mejor para ti, aquella por la cual usted estarás dispuesto a jtodo! (Perú).

c. En la competencia por el último lugar, el más peor es el de González Machi, no electo en las urnas (Paraguay).

d. ¿Lo ves? ¿Acaso no deseabas que yo, el más mejor de los caballeros andantes, viniera a socorrerte? Lo pienso apenas un instante y respondo: Bueno, pues la verdad es que no (España).

e. Soy venezolana, y me encanto esta pregunta. Lo más mejor del café es que tú te lo puedes toma[r] como quieras... (Venezuela).

f. Porque he de parecer él más peor de los humanos, Serpiente entre serpientes (México).

Por otra parte, es posible encontrar las construcciones de superlativo relativo dentro de un sintagma nominal con núcleo nominal explícito, como en los ejemplos de (3): 
(3) a. sigue jodiendo con las cifras para insistir con que vivimos la temporada de verano más mejor de la historia (Argentina).

b. La central azucarera estratosférica más mejor del mundo entero y parte de la galaxia (España).

c. Sin embargo, hallé que aunque podría darles las respuestas, la cosa más peor del mundo sería bajar corriendo intelectualmente a un hombre (México).

d. ¿Por qué siento mal? ¿Es la cosa más peor del mundo que mi novio no puede venir esta noche? (Estados Unidos).

e. se clavo un destornillador, se cayo mil veces trabajando (era el más mejor albañil que existe) con su camioneta lo atropello un tren (Estados Unidos).

En cuanto a las construcciones comparativas de superioridad ('más mejor'/ 'peor'/ 'menor que') y de inferioridad ('menos mejor'/ 'peor'/ 'menor que'), las combinaciones objeto de estudio pueden aparecer acompañadas de complemento comparativo, como sucede en los ejemplos de (4), o sin él, como se observa en (5):

(4) a. Dawn Of The Dead que, pese a quien le pese, es más mejor que la original (Argentina).

b. Es hipocresía aceptar el Terror "en determinadas circunstancias" o aceptar un "ContraTerror", o creer que el "Terror Blanco" es más mejor que el "Terror Rojo" (Perú).

c. Entonces, una chica una [sic] año más menor que yo me saludó [d]iciendo Por favor da todo de tí en el examen (Perú).

d. sobre todo, el IFE sigue siendo menos peor que el IEE (México).

(5) a. se ve espectacular, atractiva, HOT y ahora su existencia puede ser más mejor y feliz (Bolivia).

b. Además las familias viven más unidas. La vida en el campo es más mejor (Nicaragua).

c. sabes bien que me he sacrificado en momentos más peores (Colombia).

d. escribía [...] que en materia política ya habíamos tocado fondo y que cualquier cosa sería menos peor (México).

En el caso de los adverbios 'mejor' y 'peor', el contexto más frecuente es aquel en que las construcciones comparativas 'más'/ 'menos mejor' y 'más' / 'menos peor' se combinan con un verbo, fundamentalmente predicativo (6a-c), pero también con el copulativo 'estar' (6d-e):

(6) a. Cada caso se trata de forma individual. Puede que le venga bien esperar a que se sienta más mejor emocionalmente, antes de volver a intentarlo (México). 
b. Vamos a estudiar los domingos, tenemos otros ideas y después vivimos más mejor. Si alguien quiere tener su pareja ya tiene su trabajo para mantener a su niño y a su mujer (Nicaragua).

c. todas sabemos que a veces esto no es una opción. en esos casos, hay maquillajes que funcionan menos peor que otros... algunos consejos que van a ayudar: usar siempre maquillajes dermatológicamente testeados (Argentina).

d. Calladito estás más mejor. No te vistas que no vas (Venezuela).

e. el Retroblog refleja en parte la cruda verdad de nuestra sociedad: antes estábamos menos peor. El futuro nos arrolló y el pasado aún nos arrulla (Colombia).

Los adverbios 'mejor' y 'peor' también aparecen combinados con 'más' y 'menos' en un segundo tipo de contexto sintáctico-discursivo, a saber, cuando dichos adverbios modifican a un adjetivo (7a) o un participio (7b), con los que puede formar una secuencia con el artículo 'lo' (7c), como se observa en los siguientes ejemplos:

(7) a. son parte del mismo clan, lo que ocurre es que hay unos más mejor portados que otros (México).

b. Esto del clima digo yo que podrá ser más mejor calculado en zonas de temperatura constante (España).

c. las rosas arraigan lo más mejor posible si el corte tiene algunas hojas todavía asociada[s] (Honduras).

El último de los contextos en el que aparecen los adverbios 'mejor' y 'peor' modificados por 'más' y 'menos' es en construcciones comparativas con valor incidental. Este uso siempre aparece delimitado por pausas, de manera que la combinación constituye grupo fónico propio, lo que muestra su naturaleza de inciso parentético intraoracional. Estos usos sirven para completar, aclarar o afirmar la mención inmediata y tienen valor enfático, tal y como podemos comprobar en los ejemplos de (8). En efecto, en (8a) los 'rodeos' son aclarados por otro término ('aproximaciones'); en ( $8 \mathrm{~b})$ se ejemplifica y aclara, con la ponderación del adverbio 'aún', que la actuación de la otra persona no tiene sentido para la hablante.

(8) a. Aunque el tema en el que nos hemos puesto de acuerdo es el de Política y Ética, tal vez son necesarios algunos rodeos o, más mejor, aproximaciones desde puntos aparentemente distantes (Argentina).

b. no tiene ningún sentido que me uses a mí para impresionar a un grupo de hombres a los que no conozco o que me obligues a ser la cómplice involuntaria de tu actuación de validación social o, más peor aún, para transformarme en la pantalla de tu sexualidad cagona (Chile).

En suma, aunque estas combinaciones ('más'/ 'menos \{mejor/ peor/ mayor/ menor $\}^{\prime}$ ) se documentan fundamentalmente en construcciones de superlativo relativo ('la más mejor'; 'lo menos peor'), parece que no existe ninguna restricción en cuanto a 
los contextos sintáctico-discursivos en los que pueden aparecer, ya que los contextos son los mismos en los que se dan los correspondientes adjetivos y adverbios comparativos sincréticos sin cuantificador.

\subsection{La 'positivización' de los adjetivos comparativos}

En esta sección nos centraremos en presentar algunos hechos a favor de la idea de que, al igual que ha sucedido con 'mayor', los comparativos 'mejor', 'peor' y 'menor' estarían experimentando un proceso de 'positivización', es decir, en algunos contextos se pueden emplear como adjetivos positivos plenos en español actual 5 .

La primera prueba de que los comparativos sintéticos objeto de estudio pueden expresar grado positivo es que, en determinados contextos, admiten el adverbio 'muy' como modificador de grado, tal y como vemos en los ejemplos de (9), a pesar de que lo normativo es que sean modificados por 'mucho' ('mucho mejor' / 'peor'/ 'mayor'/ 'menor'), ya que contienen en su significado el cuantificador 'más' (RAE/ASALE, 2009). Como es sabido, esta es una de las principales diferencias señaladas entre los adjetivos comparativos y los adjetivos positivos. Así, Bosque (1999) indica que la forma 'mucho' se apocopa ('muy') cuando no incide sobre sintagmas comparativos: ‘*mucho alto/ mucho más alto'; 'muy alto'/ ‘*muy más alto’. Por este motivo, 'mucho’ aparece ante adjetivos comparativos sintéticos ('mucho mejor'/ '*muy mejor') ${ }^{6}$. La asimetría existente entre 'mucho mejor' y ‘*mucho superior (muy superior)' se explica, según este autor, por el hecho de que 'mejor', al ser adjetivo comparativo, “incorpora léxicamente la información semántica necesaria para que se comporte como un sintagma comparativo" (Bosque 1999: 231)7, mientras que 'superior' es un adjetivo calificativo no comparativo.

Sin embargo, como muestran los siguientes ejemplos, esta distribución en la forma del cuantificador 'mucho/ muy' no es operativa en todos los hispanohablantes.

(9) a. Tip: si quieren que el caldo tenga muy mejor sabor para tomarlo con fideos, guisquil, zanahoria y papa... pidan las pechugas con hueso (Guatemala).

b. pero lo específico que me gusta es el punto de vista. Este cuento sería muy peor si leemos esta obra con un narrador diferente (Puerto Rico).

c. a mi esposo en 11 años siempre lo trato como hijo ya que era muy menor cuando ella lo conquisto y ella trabajaba y lo mantenia hasta despues que tomo indep[e]ndencia (España).

d. la gente no se debe alarmar, es muy menor el porcentaje de gente que va a tener un hígado graso (Uruguay).

También se ha indicado que no permiten la derivación en '-mente', salvo 'mayormente' porque “el adjetivo 'mayor' admite un uso no comparativo" (Alarcos, 
1999, RAE/ASALE, 2009). De nuevo, la búsqueda en el corpus empleado para este trabajo hace posible documentar casos de los adverbios 'mejormente' (10b), 'peormente' (10c) y 'menormente' (10d), además de 'mayormente' (10a) -con el significado de 'principalmente, especialmente'-, forma que se registra en todos los países de habla hispana.

(10) a. y el coche le estará esperando para llevarle a Pelechuco. El trayecto en coche es mayormente por caminos de polvo que se encuentran en mal estado (Bolivia).

b. La vida nos pide que la acojamos, aunque ya la tenemos. Pero esa acogida es el reto: ya estamos vivos, pero necesitamos no solo estar más vivos, sino mejormente vivos. Nos desafía la vida a que la superemos (Guatemala).

c. Los efectos secundarios se agravan con la ingesta de alcohol. Peormente en pacientes que tienen problemas mentales, como trastorno bipolar, neurosis, psicosis, adicciones sea cual fuere (Ecuador).

d. desde hace quince años la organización desarrolla una importante acción social, cultural y desarrollo de programas sustentables realizados en comunidades costeras, altiplánicas y de centros menormente favorecidos (Chile).

Por último, estos adjetivos comparativos se pueden coordinar con otro adjetivo precedido del cuantificador 'más/ menos', tanto en posición inicial de la coordinación o en primer término (11a-c) como en posición final o segundo término (11d):

(11) a. tiene MUY buenos motivos para asociar el color característico del Cuerpo de Linternas Verdes y, por ende, a su más mejor y más glorioso representante pasado presente y futuro (Hal Jordan,...) (España).

b. No quería atacar tu oh espiritu núcleo y centralizador. Así que lo siento, la tuya es mucho más mejor y más larga. Yo me lo hago mirar, a ver si me extirpan este quiste (España).

c. Condenada a la soledad, esa persona dejará la comunidad donde nació para buscar un improbable futuro en otro lugar o, en el menos peor y más frecuente de los casos, engrosará las filas de la economía informal (Venezuela).

d. Realmente, para sustraerse al voy a mostrar que soy más interesante y más mejor porque tengo tal cosa de marca y tope de cara hace falta ser muy fuerte (España).

\subsection{El caso de mayor $y$ menor}

Según se ha señalado previamente, la RAE/ASALE (2005, 2017) indica que, con valor comparativo, 'mayor' tiene el significado de 'que excede a algo en cantidad o calidad' y, referido a persona, 'que excede en edad a otra'. De los cuatro adjetivos comparativos sintéticos es el único que tiene, normativamente hablando, un uso 
autorizado como adjetivo positivo en español actual, con el significado de 'entrado en años; de edad avanzada', esto es, 'adulto' o 'anciano' (Madero Kondrat, 1983, para el caso del español de México, y Vigara Tauste, 2010, 2011). Por su parte, Bosque (1999) explica el par 'mucho mayor/ muy mayor' indicando que en el primer caso 'mayor' es comparativo, mientras que en el segundo se trata de un adjetivo calificativo simple que posee el significado de 'anciano' o 'crecido'. En este sentido, la RAE/ASALE (2009) menciona que en el español coloquial europeo (y pocas veces en el americano) 'mayor' se emplea -siempre con referencia a la edad- como adjetivo positivo, por lo que las combinaciones 'más mayor' y 'muy mayor' no resultan incorrectas allí donde se emplean.

Uno de los pocos trabajos realizados sobre este adjetivo, y en concreto sobre la combinación 'más mayor', es el de Vigara Tauste (2010, 2011). Esta autora considera que la construcción 'más mayor' es el resultado de la lexicalización de 'mayor' como adjetivo positivo cuando este adjetivo posee significado temporal, empleo generalizado en España y conocido en Hispanoamérica (Vigara Tauste, 2010). La autora compara la situación existente en español con la que se da en otras lenguas romances y constata una serie de hechos que distinguen el caso del español. Así, Vigara Tauste (2011) señala que el adjetivo se ha conservado en francés (majeur) solo para 'mayor de edad' y en portugués (maior) para indicar dimensión física ('más alto'). En cambio, el adjetivo empleado de forma general para referirse a la edad es vecchio en italiano, vieux en francés y velho en portugués, mientras que en el habla infantil se emplea grande en italiano y grand en francés. Así pues, el empleo de 'mayor' como adjetivo positivo de significado temporal sería una característica exclusiva del español, en concreto, del español de España, pues, aunque se conoce y se emplea con ese sentido temporal también en Hispanoamérica, es 'grande' el adjetivo que se usa para referirse a la edad y el paso del tiempo en Hispanoamérica, especialmente en Argentina y en Uruguay (Vigara Tauste, 2011).

Sin embargo, los datos obtenidos en el corpus muestran una serie de hechos interesantes que no han sido previamente revisados. En concreto, hemos documentado el empleo de 'mayor' como adjetivo positivo también cuando este adjetivo presenta un significado distinto del temporal, esto es, cuando su significado no se relaciona con el ámbito de la edad. Así se observa en los ejemplos de (12), en los que la construcción 'más mayor' incide sobre sustantivos inanimados, de manera que se da un significado de dimensión física literal, como en (12a-c), o figurado, referido a una posición elevada en una escala, como en (12d-f):

(12) a. además encuentro que hacen que la mano se vea más mayor (Chile).

b. la Tierra tuvo dos lunas, siendo la más pequeña absorbida por la más mayor por una colisión descomunal (España). 
c. Quién sabe si estamos sentados sobre los más mayores yacimientos de cobre después de los de Chile (Panamá).

d. De los alimentos estudiados, las papas fritas fueron los que tuvieron el más mayor nivel de acrilamida (Colombia).

e. es grato e intenso, pero el recuerdo del espíritu se percibe con más mayor intensidad en el goce del momento (Chile).

f. se ha expresado con antelación que tanto la RAAN como la RAAS tienen los más mayores índices de mortalidad (Nicaragua).

Además, hemos documentado algunos ejemplos en los que aparece la expresión 'cada más mayor' (en un uso de 'cada' pronominal, similar al 'cada' que propio de México, los países centroamericanos, el caribe continental y Paraguay, RAE/ASALE, 2009) y 'cada vez más mayor', como los que se muestran en (13), que pueden estar sujetos a un doble análisis: [[cada vez más] mayor] vs. [[cada vez] más mayor]. En este segundo caso, nos encontraríamos -al igual que en los ejemplos de (12)- con la expresión 'más mayor' en contextos en los que 'mayor' no presenta significado temporal, sino de dimensión:

(13) a. Y, eso significa reconocer que la competencia es cada vez más mayor en este mundo tecnológico y globalizado (España).

b. Los ciudadanos tienen cada vez más mayor conciencia de esto (Argentina).

c. La actuación de los hackers es cada vez más mayor en el mundo (Honduras).

d. la probabilidad de que tenga o padezca demencia es cada más mayor (México).

e. y si bien la extensión de luz es cada más mayor, seguimos y seguiremos teniendo espacios donde esa luz no llega (Estados Unidos).

Así pues, los ejemplos documentados apuntan hacia el hecho de que el proceso de lexicalización de 'mayor' como adjetivo positivo, ya culminado cuando este adjetivo posee significado temporal, se estaría extendiendo a los casos en los que 'mayor' presenta otros significados, como son el de dimensión física real o figurada. Se trata de un fenómeno que no se había mencionado en la bibliografía previa sobre el tema.

Por lo que respecta a 'menor', el Diccionario panhispánico de dudas (RAE/ASALE, 2005) señala que entre los usos no comparativos de 'menor' se encuentra aquel en el que este adjetivo es sinónimo de 'pequeño'. Por su parte, el Diccionario de la lengua española (RAE/ASALE, 2017) ofrece el significado de 'que es inferior a otra cosa en cantidad, intensidad o calidad' o que es 'menos importante con relación a algo del mismo género'. Referido a persona, significa 'que tiene menos edad que otra'. Los ejemplos de (14) ofrecen casos concretos de este empleo de 'menor' como adjetivo temporal. El hecho de que se combine con los adverbios comparativos 'más' y 
'menos' indica que se encuentra en proceso de positivación, de forma similar a lo sucedido con 'mayor'. Por tanto, en español actual, 'menor' también se emplea en el campo de la edad.

(14) a. Cuando estaba en la secundaria, San Valentín cayó el mismo día que un examen. Entonces, una chica una [sic] año más menor que yo me saludó siciendo [sic] "Por favor da todo de tí en el examen" y me dio un chocolate (Perú).

b. El más menor de sus hijos, Ever Villalta se graduó el 17 de mayo del presente año de la Universidad de George Mason en Virginia en donde obtuvo una licenciatura en gobierno y política internacional (El Salvador).

c. Andaría con alguien de hasta... 50 años (eso ya depende de otras cosas) No andaría con alguien de menos de... Pues creo que de mi edad, no estoy acostumbrada a los de mi edad y menos menores, también depende de la persona en particular (México).

A ejemplos como los de (14), en los que 'menor' presenta un significado temporal, se suman otros como los de (15), en los que este adjetivo no posee un significado relacionado con la edad, sino con la dimensión física, como hemos visto que sucede también en el caso de 'mayor'. De hecho, el Diccionario panbispánico de dudas (RAE/ASALE, 2005) ofrece otros usos no comparativos de 'menor', además de aquel en el que este adjetivo presenta significado temporal: a) con el significado 'de importancia o categoría secundarias' (poetas menores) (15b, 15c); b) como equivalente de 'ninguno' en oraciones negativas, precedido de artículo y antepuesto al sustantivo ('No hice el menor comentario’) (15a).

(15) a. aguanto una caída normal de una escalera de cemento sin presentar ni la más menor marca o rayón (Paraguay).

b. Todos los cambios, por más menores que fueran, son justificados (Argentina).

c. La prensa hizo muy popular aquella operación y estoy orgulloso de haber participado en ella. También he cometido otro tipo de delitos más menores. He desvalijado casas enteras, como la mansión de esos ricachuelos llamados Temperton (México).

En otras lenguas romances como el francés las formas majeur y mineur han perdido su valor comparativo y ya no significan 'más grande' y 'más pequeño' (Académie française, 2015). Volveremos a este hecho en las consideraciones finales.

\subsection{El caso de mejor $y$ peor}

Como venimos señalando a lo largo de este trabajo, en las obras de referencia consultadas, de entre los cuatro comparativos sincréticos heredados del latín, se acepta 
únicamente el empleo de 'mayor' como adjetivo positivo circunscrito al ámbito temporal o de la edad, mientras que esta posibilidad no se reconoce para el resto. Así, por ejemplo, Vigara Tauste (2011: 125) afirma que la construcción temporal 'más mayor" sigue su curso "al margen del resto de los adjetivos comparativos heredados del latín ('menor', 'mejor' y 'peor'), que conservan su función -salvo excepciones también- y su significado original (con la excepción de la lexicalización de 'menor' [de edad])".

Sin embargo, según se ha mostrado en el apartado 1, 'mejor' y 'peor' también se documentan en construcciones comparativas analíticas ('más'/ 'menos mejor'/ 'mejores'/ 'peor'/ 'peores'). En concreto, como se mostró previamente, 'mejor' se registra sobre todo combinado con 'más' y 'peor' combinado con 'menos'. Así, de los 363 casos registrados de 'más' + comparativo sintético en singular (cf. Tabla 1), 280 corresponden a 'más mejor' (77,13\%); y de los 78 casos de 'más' + comparativo sintético en plural (cf. Tabla 2), 58 constituyen casos de 'más mejores' (74,36 \%). Por su parte, de los 433 casos documentados de 'menos' + comparativo sintético en singular (cf. Tabla 3), 406 corresponden a 'menos peor' (93,76\%); y de los 38 casos de 'menos' + comparativo sintético en plural (cf. Tabla 4), 31 corresponden a 'menos peores’ $(81,58 \%)$.

En (16) ofrecemos algunos de los ejemplos de 'más mejor' / 'mejores'. En algunos casos, el empleo de esta combinación aparece marcada entre comillas o con algún comentario que pone de manifiesto que el hablante está haciendo un uso consciente de ella, como se observa en (16c). Por otra parte, varios de los ejemplos corresponden a la expresión 'mi más mejor amigo', esto es, precedidos de un posesivo, como vemos en (16d).

(16) a. Algunos dirán que ayuda a saber cuál es el más mejor o el menos malo (Chile).

b. Después de pasar su infancia ayudando a su padre, uno de los más mejores ladrones de arte del mundo, Katarina Bishop intenta dejar el negocio familiar (España).

c. Aún recuerdo a más de una pensando que cuanto más grandes, 'más mejor', y si los combinabas con unas mechas horrendas y tintes imposibles, MEJOR (España).

d. sólo por las carcajadas que me habéis despertado hoy, ya sois mis más mejores amigos de la red (España).

Con todo, ejemplos como el de (16d) son minoritarios, frente a los numerosos casos en que 'más mejor'/ 'mejores' parece emplearse de forma natural con el significado de 'más bueno(s)' o 'más bien'. Así pues, los datos registrados en el corpus muestran que el comparativo sincrético 'mejor' se estaría lexicalizando como adjetivo 
y adverbio positivo en español actual, con el significado de 'bueno' y 'bien', respectivamente.

En lo que respecta a 'menos peor' / 'peores', podemos observar en los ejemplos de (17) cómo 'peor' se emplea con el significado de 'malo/ mal', esto es, como adjetivo $(17 \mathrm{a}-\mathrm{c})$ o adverbio positivo $(17 \mathrm{~d})$ :

(17) a. Yo me he fijado que en las últimas elecciones muchos votan por el menos peor (Chile).

b. Realmente fue menos peor de lo que me había imaginado en ese momento en el que sonó la puerta (Colombia).

c. Tarde o temprano, lo que se termina provocando es, en el menos peor de los escenarios, la hipocresía y las vidas basadas en mentiras (Guatemala).

d. En todo caso, que bien por Colunga que haya medio evolucionado a hacerlo menos peor en las siguientes telenovelas (España).

Con frecuencia, este uso aparece en contextos relacionados con la política o, en general, cuando se habla de elegir entre varias opciones, ninguna de las cuales se considera deseable, según se aprecia en los ejemplos anteriores. Así las cosas, consideramos que el comparativo sincrético 'peor' está experimentando un incipiente proceso de lexicalización como adjetivo y adverbio positivo, limitado por el momento a un contexto discursivo restringido. Este hecho puede explicar que se documenten muchos más casos de 'menos peor'/ 'peores' (83,24\%, 437 casos de 525, suma de las 'Tablas 3 y 4) que de 'más peor'/ 'peores' (16,76\%, 88 casos de 525, suma de las Tablas 1 y 2$)$.

\section{CONCLUSIONES}

En este trabajo hemos revisado la aparición de los comparativos sintéticos 'mejor', 'peor', 'mayor' y 'menor' en construcciones comparativas con los adverbios 'más' y 'menos'. A partir de los datos procedentes del Corpus del español: Web/Dialectos, hemos mostrado que la combinación de 'más' y 'menos' con un comparativo sintético no se limita al caso de 'mayor', fenómeno habitualmente tratado en la bibliografía y aceptado desde el punto de vista normativo, sino que se da también con los otros tres comparativos sintéticos.

En concreto, los datos analizados nos permiten confirmar que estas combinaciones -documentadas desde antiguo- son más abundantes en singular (87,28\%, 796/912) que en plural $(12,72 \%, 116 / 912)$, y que, dentro de los cuatro comparativos sintéticos, son los casos de 'más mejor' $(77,13 \%)$ y 'menos peor' $(93,76 \%)$ los que alcanzan los porcentajes más elevados.

Los datos presentados confirman asimismo que no se trata de un uso exclusivo de España y documentado 'raramente en América', como señala la RAE/ASALE (2009), 
sino que goza de plena autonomía en las variedades americanas, como se ha visto en detalle en las Tablas 1-4.

Dos son los contextos sintáctico-discursivos fundamentales en los que aparecen estas combinaciones: i) en construcciones de superlativo relativo, con coda o sin ella ('la más mejor de todas'/ 'la más mejor'), y con núcleo nominal explícito o sin él ('la mujer más mejor del mundo'/ 'la más mejor'); y ii) en comparativas de superioridad e inferioridad (con o sin complemento comparativo). En cuanto a los adverbios 'mejor' y 'peor', se suelen combinar con un verbo predicativo ('vivimos más mejor') o con el copulativo 'estar' ('estábamos menos peor'), pero también aparecen modificando a un adjetivo ('más mejor calculado') y en construcciones de valor incidental (entre pausas).

Por otro lado, hemos ofrecido pruebas evidentes del proceso de 'positivización' que estarían experimentando en español actual las cuatro formas: admiten el modificador de grado 'muy' ('muy mayor'/ 'menor' / 'mejor' / 'peor'), la derivación en '-mente' ('mejormente', 'peormente', 'mayormente', 'menormente') y la coordinación con otro adjetivo en construcción comparativa ('más mejor y más larga'). Además, hemos visto que la lexicalización de 'mayor' como adjetivo positivo no solo se documenta en el español de España y con el significado temporal ('persona más mayor'), sino que también se registra ampliamente en América y con un significado de dimensión física real o figurada ('los más mayores yacimientos de cobre'; 'con más mayor intensidad'; 'cada (vez) más mayor'). Lo mismo encontramos para el caso de 'menor', que presenta un sentido temporal ('el más menor de sus hijos') y otro de dimensión física ('delitos más menores'). Por último, en cuanto a las formas 'mejor' y 'peor', la primera se estaría lexicalizando con el significado de 'más bueno/ bien' ('los más mejores ladrones'; 'ya está más mejor de su enfermedad'; 'vos podés hacerlo más mejor') y la segunda con el de 'más malo/ mal' ('fue menos peor'; 'el menos peor de los escenarios').

Con todo, creemos que el fenómeno aquí analizado es una prueba de que el sistema excepcional de gradación comparativa con adjetivos y adverbios en -or está en 'reestructuración', en el sentido de que algunos hablantes modifican la semántica de los comparativos para hacerlos positivos, por lo que podrían llegar a perderse en un futuro, tal y como sucede en inglés (more better 'más mejor' y less worse 'menos peor'). Se trata de un fenómeno que se documenta también en otras lenguas romances. Así, en portugués tenemos mais melhor ('más mejor') y menos pior ('menos peor') (18a); en catalán, més millor ('más mejor') y menys pitjor ('menos peor') (18b); en francés, plus meilleur ('más mejor'), plus mieux ('más mejor'), moins pire ('menos peor') y moins pis ('menos peor') ${ }^{8}$ (18c); finalmente, en italiano, più migliore ('más mejor') y meno peggio ('menos peor') (18d), tal y como muestran los siguientes ejemplos obtenidos en la red social Twitter, usos no normativos, en los que mantenemos la grafía original. 
(18) a. eu só queria ser mais melhor nesse jogo.

(Yo solo quería ser más mejor en este juego).

b. Ah, perdona, no t'entés En el que al Barça es refereix, sens dubte, Schuster més millor que Maradona.

(Ah, perdona, no te he entendido. En lo que al Barça se refiere, sin duda, Schuster más mejor que Maradona).

c. Selon moi l'éducation francophone est plus mieux que l'éducation anglophone.

(En mi opinión, la educación francófona es más mejor que la educación anglófona).

d. Sei la più migliore.

(Eres la más mejor).

\section{REFERENCIAS BIBLIOGRÁFICAS}

Académie française (2015). Plus pire, moins pire, Dire, ne pas dire. París: Académie française [en línea]. Disponible en: http://www.academie-francaise.fr

Alarcos, E. (1999). Gramática de la lengua española. Madrid: Espasa.

Bello, A. (1847). Gramática de la lengua castellana, destinada al uso de los americanos. Santiago de Chile: Imprenta del Progreso.

Bosque, I. (1999). El sintagma adjetival. Modificadores y complementos del adjetivo. Adjetivo y participio. En I. Bosque \& V. Demonte (Dirs.), Gramática descriptiva de la lengua española (pp. 217-310). Madrid: Espasa-Calpe.

Covarrubias, S. (1611). Tesoro de la lengua castellana o española. Barcelona: Horta.

Felíu Arquiola, E. \& Pato, E. (2020). En torno a la denominada concordancia adverbial en español: Tres casos de variación. Madrid: CSIC, Anejos de la Revista de Filología Española.

García de Diego, V. (1970). Gramática histórica española. Madrid: Gredos.

Gómez Torrego, L. (1998). Gramática didáctica del español. Madrid: SM.

Grandgent, Ch. H. (1970). Introducción al latín vulgar. Madrid: CSIC.

Kany, Ch. (1969). Sintaxis hispanoamericana. Madrid: Gredos.

Madero Kondrat, M. (1983). La gradación del adjetivo en el habla culta de la ciudad de México. Anuario de Letras, 21, 71-118.

Meyer-Lübke, W. (1900). Grammaire des langues romanes, III. Sintaxe. París: Welter. 
Pato, E. \& Viejo Fernández, X. (2017). Mu(n)cho guapa y mu(n)cho bien: Mu(n)cho como cuantificador 'pleno' de adjetivos y adverbios en castellano y en asturiano. Bulletin of Spanish Studies, 94(1), 1-23.

Piera, C. \& Varela, S. (1999). Relaciones entre morfología y sintaxis. En I. Bosque \& V. Demonte (Dirs.), Gramática descriptiva de la lengua española (pp. 4367-4422). Madrid: Espasa-Calpe.

Real Academia Española. Banco de datos CORDE. Corpus diacrónico del español. Madrid: RAE [en línea]. Disponible en: $\underline{w w w . r a e . e s}$

Real Academia Española \& Asociación de Academias de la Lengua Española (2005). Diccionario panhispánico de dudas. Madrid: Santillana.

Real Academia Española \& Asociación de Academias de la Lengua Española (2009). Nueva gramática de la lengua española. Madrid: Espasa.

Real Academia Española \& Asociación de Academias de la Lengua Española (2017). Diccionario de la lengua española. Edición del Tricentenario. Madrid: RAE.

Robles Ávila, S. (1998). Análisis de errores: El caso de los comparativos y superlativos del español. En F. Moreno Fernández et al. (Eds.), Actas del VIII Congreso Internacional de la ASELE. La Enseñanza del Español como Lengua Extranjera: del Pasado al Futuro (pp. 685-692). Alcalá de Henares: Universidad de Alcalá.

Rodríguez Molina, J. \& Octavio de Toledo y Huerta, Á. (2017). La imprescindible distinción entre texto y testimonio: El CORDE y los criterios de fiabilidad lingüística. Scriptum Digital, 6, 5-68.

Romero Cambrón, Á. (1998). Historia sintáctica de las construcciones comparativas de desigualdad. Cáceres: Universidad de Castilla-La Mancha.

Seco, M. (2000). Diccionario de dudas y dificultades de la lengua española. Madrid: EspasaCalpe.

Seco, M. \& Hernández, E. (1999). Guia práctica del español actual. Diccionario breve de dudas y dificultades. Madrid: Espasa-Calpe.

Vigara Tauste, A. M. (2010). Gramática, excepción, norma y uso: A propósito de la construcción más mayor. Aspectos sincrónicos y diacrónicos (I). Revista Española de Lingüistica, 40(2), 125-140.

Vigara Tauste, A. M. (2011). Gramática, excepción, norma y uso: A propósito de la construcción más mayor. Aspectos sincrónicos y diacrónicos (II). Revista Española de Lingüistica, 41(1), 113-127. 


\section{NOTAS}

1 Son adjetivos y adverbios comparativos 'mejor' ('más bueno' y 'más bien') y 'peor' ('más malo' y 'más mal'), mientras que 'mayor' ('más grande') y 'menor' ('más pequeño') son solo adjetivos comparativos (RAE/ASALE, 2009: 3374).

2 Según los datos del Corpus del español: Web/Dialectos, la forma 'mayor' se registra 1244974 veces (1 013057 en singular y 231917 en plural), 'menor' 411396 (274 227 en singular y 137169 en plural), 'mejor' 1962073 (1 606166 en singular y 355907 en plural) y 'peor' 296 166 (251 614 en singular y 44552 en plural).

${ }^{3}$ La prueba Anova (o Análisis de varianza) compara varios grupos en una variable cuantitativa, en nuestro caso la variable 'adjetivo comparativo' con el factor 'país'. La F refleja el grado de similitud entre las medias comparadas y el valor de $p$ (establecido en 0,05) representa la probabilidad de que la diferencia se deba o no al azar.

${ }^{4} \mathrm{Y}$ a adverbio positivo en el caso de 'mejor' y 'peor'. En aras de una mayor sencillez expositiva, en ocasiones emplearemos de forma general el término 'adjetivo' para englobar las cuatro formas objeto de estudio ('mejor', 'peor', 'mayor', 'menor'), lo que no significa que olvidemos que los dos primeros comparativos sintéticos pueden funcionar como adjetivos y como adverbios.

5 Se ha establecido que estos adjetivos son invariables en género, por lo que las formas '*mayora', 'menora', 'peora' y 'mejora' no serían posibles. Sí lo son, en cambio, como sustantivos: 'mayora' ('mujer del mayor') y 'mejora' ('medra, adelantamiento y aumento de algo') (RAE/ASALE, 2017). Sin embargo, una búsqueda en el corpus empleado, y en redes sociales como Twitter, permite documentar casos de 'mayora' ('una resolución mucho mayora', Argentina), 'menora' ('yo tengo 33 y Joy es tres años y medio 'menora”, México), 'peora' ('refleja una situación peora', Ecuador) y 'mejora' ('cualquier cosa es mejora que esa porqueria de pagina oficial', Colombia), lo que muestra que para algunos hablantes, especialmente adolescentes y jóvenes adultos, estas formas son significativas y variables en términos morfológicos.

6 Para el caso de 'mucho' como cuantificador de adjetivos ('mucho guapa') y adverbios ('mucho bien') véase Pato y Viejo Fernández (2017). Para la 'concordancia adverbial' de esta forma véase Felíu Arquiola y Pato (2020).

${ }^{7}$ Por su parte, Piera y Varela (1999: 4398) consideran que los comparativos sintéticos 'mejor' y 'peor' son palabras policategoriales, esto es, palabras que "tanto formal como semánticamente equivalen a dos categorías sucesivas distintas". En el caso de los comparativos sintéticos, se trataría de adjetivos o adverbios al tiempo que de sintagmas cuantificativos.

${ }^{8}$ La Académie française (2015) indica que "Si, en dehors du langage des tout jeunes enfants, on n'entend guère plus mieux, les locutions plus pire et moins pire, elles, se répandent de plus en plus chez des locuteurs de tous âges. On rappellera que ces formes signifiant «plus plus mauvais» et «moins plus mauvais» sont de graves incorrections" (http://www.academiefrancaise.fr/plus-pire-moins-pire). 